\title{
A Musical Instrument for Facilitating Musical Expressions
}

\author{
Kazushi Nishimoto \\ Japan Advanced Institute of Science and \\ Technology, Hokuriku \\ PRESTO, JST \\ 1-1, Asahidai, Tatsunokuchi, Nomi, Ishikawa \\ 923-1292, Japan \\ +81761511812 \\ knishi@acm.org
}

\author{
Chika Oshima, Yohei Miyagawa, \\ Takashi Shirosaki \\ Japan Advanced Institute of Science and \\ Technology, Hokuriku \\ 1-1, Asahidai, Tatsunokuchi, Nomi, Ishikawa \\ 923-1292, Japan \\ +81761511812 \\ \{cooshi, y-miyaga, t-shiro\}@jaist.ac.jp
}

\begin{abstract}
In this paper, we propose a new musical instrument that allows people to concentrate on controlling indiscrete elements so that they can directly create their musical expressions. We describe a prototype musical instrument and demonstrate two applications of the prototype to show its effectiveness.
\end{abstract}

\section{Keywords}

Musical expressions, discrete/indiscrete elements

\section{INTRODUCTION}

Conventional music consists of two kinds of elements, i.e., discrete elements and indiscrete elements. The pitch of each note is the discrete element, while tempo, rhythm, "agogik," "dynamik," and so on are the indiscrete elements. By integrating both types of elements, an entire piece of music is constructed. Therefore, a composer carefully considers both types of elements and records them as the score of the piece, while a performer controls both types of elements when performing on a musical instrument so that $\mathrm{s} /$ he can create her/his musical expression for the piece.

However, the discrete element has a different importance for the composer than for the performer. Namely, the composer has complete freedom of choice in the pitches of the notes in a composition, which allows the composer to fully exhibit his/her creativity. However, the performer basically does not have the freedom to select the pitches of the notes. While the performer has some degree of freedom in selecting the pitches of the notes along with the directed pitches in a score for popular music (ad-lib performance in Jazz or Rock music, in particular), s/he must completely obey the composer's directions on pitches for classical music. Though the performer must input the discrete type of elements concurrently, it is not essential for his/her expression. Hence, we can say that the performer can express his/her musical expression by using only the indiscrete type of elements.

Accordingly, the performer is obliged to input the pitches simply because the conventional musical instruments require the pitch data of the notes. Accurate reproduction of the pitches of notes as directed by the composer forces the performer to make a strong but non-creative effort. The performer wastes a significant amount of his/her cognitive ability on this effort. On the other hand, for the novice, accurate reproduction of the sequence of pitches is a formidable initial barrier to performing the musical instrument. This barrier often leads the novice to giving up the idea of mastering the musical instrument.

Consequently, we propose a new musical instrument that releases people from the requirement of inputting the discrete element and that allows them to concentrate on control of the indiscrete elements. As a result, they can directly create their musical expressions. In this paper, we illustrate the prototype musical instrument named "Coloring-in Piano (CiP)" and demonstrate two applications of the CiP to show its effectiveness.

\section{PROTOTYPE MUSICAL INSTRUMENT: CiP}

Figure 1 shows the structure of CiP. We used a Yamaha silent grand piano $\mathrm{C} 5$ professional model that outputs MIDI (Musical Instrument Digital Interface) note-on/off and pedal control messages. The piano is connected to an SGI Indy workstation. Before performing, it is necessary to feed the sequence of MIDI note-numbers (corresponding to the pitches) of the piece into the Indy. While performing, the

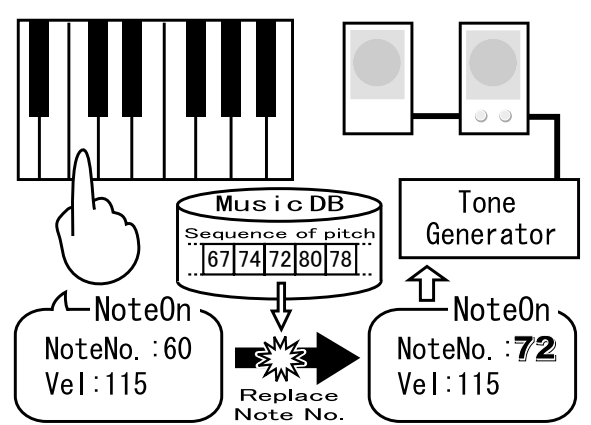

Figure 1. Structure of CiP 
Indy replaces the fed note-numbers with the played notenumbers in order. Finally, the Indy outputs the replaced pitch numbers and inputs them into the sound generator. Accordingly, CiP always outputs the correct pitch sound by touching any key. On the other hand, note-on (key down) velocity, note-off (key up) velocity, onset/offset timing, and pedal messages are output as the performer plays.

Therefore, in a performance with $\mathrm{CiP}$, the computer is responsible for controlling the discrete element, while the performer is responsible to control the indiscrete elements. This division of labor in musical performance enables freedom in the performer's creative musical expression.

\section{APPLICATIONS OF CiP}

\section{Supporting Piano Lessons}

A piano lesson is a place where a teacher cultivates pupils' musical creativity. The teacher attempts to convey his/her knowledge of piano performance in the lesson. A model performance is a typical way to show the teacher's way of expression. However, if a pupil studies a highly technical piece that the teacher has seldom or never performed, the teacher may not be able to perform it perfectly at once. Even in that case, the teacher has knowledge of how to perform it. In such a case, CiP would surely be useful. By using $\mathrm{CiP}$, the teacher would be able to immediately show his/her expressions in playing the piece without worrying about accurate control of the pitches of the notes.

We compared the similarity and the musicality between performances with $\mathrm{CiP}$ and performances with a conventional piano by subjective evaluation. Moreover, the performance data were analyzed. These results indicated that a performer can play with $\mathrm{CiP}$ as musically as playing with a conventional piano. In addition, one of the authors, who is a piano instructor, played technically unfamiliar pieces with CiP and a conventional piano. CiP could support performance of technical passages and the parts of a piece involving difficult articulations by using only two fingers and/or five fingers, and the performer could expressively play pieces that she had seldom/never performed. Consequently, CiP can facilitate the teacher's ability to convey musical knowledge to the pupil.

\section{Supporting Composition MIDI Sequence Data}

It has recently become possible for people to perform any musical piece by using a computer. When MIDI sequence data are input, the computer can perform even a symphony by a full orchestra. This benefits those who want to perform music but cannot play any musical instrument.

There are two ways of composing MIDI sequence data, i.e., a real-time input method and a step-by-step input method. In the real-time input method, the sequence data are basically composed by performing on a musical instrument that outputs MIDI data in the usual manner. Therefore, it is impossible for people who cannot play any musical instrument to compose MIDI sequence data by the realtime input method. In the step-by-step input method, people compose MIDI sequence data by inputting pitch, velocity, onset time, and offset time of each note as numerical values.
Therefore, anyone can compose MIDI sequence data by the step-by-step input method even if s/he cannot play any musical instrument. However, it is a very troublesome task to achieve rich musical expressions by the step-by-step input method. Accordingly, the quality of the composed MIDI sequence data becomes musically poor, or people eventually give up the idea of composing computer music.

Using CiP, this situation can be dramatically improved. We proposed a "two-step" input method. In this method, only the sequence of pitch is input in the first step, without giving consideration to the velocity and the onset/offset time data. Then, in the second step, the expressions of the piece, i.e., the velocity and the onset/offset time data, are input with $\mathrm{CiP}$.

We conducted experiments using subjects as performers to compare the two-step input method with the two conventional two input methods. As a result, we found that people who cannot play a piano could create sufficiently musically rich MIDI sequence data by the two-step input method, while they could create only poor MIDI sequence data by the conventional methods.

\section{RELATED WORKS}

Various supporting systems for musical performance have been proposed, and some of them are now commercially available. "Two Finger Piano" [1] is a toy system that allows the user to coarsely handle tempo and dynamik for each "beat" (not for each note) by using two fingers. However, it is impossible to fully control musical expressions by the beat-level performance. "Lighted Keyboard" [2] is equipped with the "3-Step Teaching System" to support self-study of a piano. The first teaching step provides a quite similar function to CiP in outputting available pitches by hitting any key. However, this system always outputs the constant velocity value. Thus, such ordinary systems can only be applied for novices' amusement, while our new musical instrument aims at facilitating pure musical expression not only by novices but also by professionals.

\section{CONCLUSIONS}

We proposed a new musical instrument that releases the performer from the non-creative effort of controlling the discrete musical elements, i.e., the pitches. Using this instrument, the performer can concentrate only on the control of the indiscrete musical elements that are essential for musical expression in musical performance. Through the experiments on using $\mathrm{CiP}$, our prototype instrument, we confirmed that this new musical instrument allows people, not only professionals but also novices, to directly exhibit their rich expressions in musical performance.

\section{REFERENCES}

1. Takeuchi, Y., and Katayose.H., Representation of Music Expression with Two Finger Piano, IPSJ SIGNotes Music and Computer, No.011-006, 1995.

2. CASIO Web-page of Lighted Keyboards. Available at http://www.casio.com/musicalinstruments/section.cfm?s ection $=35$ 